\title{
Cortical Excitability Measured with nTMS and MEG during Stroke Recovery
}

\author{
Jyrki P. Mäkelä, ${ }^{1}$ Pantelis Lioumis, ${ }^{1,2}$ Kristina Laaksonen, ${ }^{3,4}$ Nina Forss, ${ }^{3,4}$ \\ Turgut Tatlisumak, ${ }^{3,5,6}$ Markku Kaste, ${ }^{3}$ and Satu Mustanoja ${ }^{3}$
}

\author{
${ }^{1}$ BioMag Laboratory, HUS Medical Imaging Center, Helsinki University Hospital and University of Helsinki, 00029 Helsinki, Finland \\ ${ }^{2}$ Neuroscience Center, University of Helsinki, 00014 Helsinki, Finland \\ ${ }^{3}$ Department of Neurology, Helsinki University Hospital and University of Helsinki, 00029 Helsinki, Finland \\ ${ }^{4}$ Department of Neuroscience and Biomedical Engineering, Aalto University School of Science, P.O. Box 15100, 00076 Espoo, Finland \\ ${ }^{5}$ Institute of Neuroscience and Physiology, Sahlgrenska Academy, University of Gothenburg, 41345 Gothenburg, Sweden \\ ${ }^{6}$ Department of Neurology, Sahlgrenska University Hospital, 41345 Gothenburg, Sweden
}

Correspondence should be addressed to Jyrki P. Mäkelä; jyrki.makela@hus.fi

Received 27 February 2015; Accepted 5 May 2015

Academic Editor: Yong Jeong

Copyright (C) 2015 Jyrki P. Mäkelä et al. This is an open access article distributed under the Creative Commons Attribution License, which permits unrestricted use, distribution, and reproduction in any medium, provided the original work is properly cited.

Objective. Stroke alters cortical excitability both in the lesioned and in the nonlesioned hemisphere. Stroke recovery has been studied using transcranial magnetic stimulation (TMS). Spontaneous brain oscillations and somatosensory evoked fields (SEFs) measured by magnetoencephalography (MEG) are modified in stroke patients during recovery. Methods. We recorded SEFs and spontaneous MEG activity and motor threshold (MT) short intracortical inhibition (SICI) and intracortical facilitation (ICF) with navigated TMS (nTMS) at one and three months after first-ever hemispheric ischemic strokes. Changes of MEG and nTMS parameters attributed to gamma-aminobutyrate and glutamate transmission were compared. Results. ICF correlated with the strength and extent of SEF source areas depicted by MEG at three months. The nTMS MT and event-related desynchronization (ERD) of beta-band MEG activity and SICI and the beta-band MEG event-related synchronization (ERS) were correlated, but less strongly. Conclusions. This first report using sequential nTMS and MEG in stroke recovery found intra- and interhemispheric correlations of nTMS and MEG estimates of cortical excitability. ICF and SEF parameters, MT and the ERD of the lesioned hemisphere, and SICI and ERS of the nonlesioned hemisphere were correlated. Covarying excitability in the lesioned and nonlesioned hemispheres emphasizes the importance of the hemispheric balance of the excitability of the sensorimotor system.

\section{Introduction}

The motor system is a dynamic network of cortical and subcortical areas interacting through excitatory and inhibitory circuits, modulated by somatosensory input. The network balance is disturbed after stroke [1-3]. Modifications of cortical excitability enable recovery and reorganization of the remaining motor areas both in animal models $[4,5]$ and in humans $[1,6]$. Transcranial magnetic stimulation (TMS) and magnetoencephalography (MEG) have both been applied in stroke patients to reveal cortical excitability changes.

Motor threshold (MT), that is, the minimal TMS intensity eliciting motor evoked potentials (MEPs), is related to membrane excitability as it is influenced by drugs affecting neuronal ion channels. Paired pulse TMS (PP-TMS) reveals short-interval intracortical inhibition (SICI), related to the activation of GABA-A receptors and intracortical facilitation (ICF) attributed mainly to glutamatergic NMDA receptor activation (for references, see [7]). In acute stroke, the MT is increased in the lesioned hemisphere $(\mathrm{LH})$ whereas in the nonlesioned $(\mathrm{NH})$ hemisphere it is normal [8] or decreased one month after stroke [9]. SICI is decreased in both hemispheres early after stroke; ICF is stronger in $\mathrm{NH}$ in severe than mild strokes $[1,6]$.

Finger movements and median nerve [10] or finger stimulation [11] modify spontaneous MEG oscillations over the sensorimotor cortex in the beta band $(15-25 \mathrm{~Hz})$. They are suppressed at $100-300 \mathrm{~ms}$ after tactile stimulation 
TABLE 1: Patient demographics and behavioral scores.

\begin{tabular}{|c|c|c|c|c|c|c|c|c|c|c|c|c|c|}
\hline $\mathrm{Pt}$ & Sex & Age & Les. site & Hemi & Les. class & Les. size & NIHSS1 & NIHSS2 & $\mathrm{mRs} 1$ & mRs2 & BI1 & BI2 & Sense loss \\
\hline 1 & $\mathrm{M}$ & 60 & Hand MCx & $\mathrm{R}$ & $\mathrm{C}$ & 0.1 & 0 & 0 & 2 & 1 & 100 & 100 & $-{ }^{\prime}$ \\
\hline 2 & $\mathrm{~F}$ & 68 & GP & $\mathrm{L}$ & SC & 1 & 2 & 2 & 1 & 1 & 100 & 100 & $t^{\prime}$ \\
\hline 3 & $\mathrm{~F}$ & 72 & Primary MCx & $\mathrm{L}$ & $\mathrm{C}$ & 0.3 & 0 & 0 & 1 & 1 & 100 & 100 & $-^{\prime}$ \\
\hline 4 & F & 46 & BGp & $\mathrm{L}$ & $\mathrm{C}+\mathrm{SC}$ & 70 & 8 & 6 & 3 & 3 & 65 & 85 & $+^{\prime}$ \\
\hline 5 & $\mathrm{M}$ & 60 & MCA territory & $\mathrm{L}$ & $\mathrm{C}+\mathrm{SC}$ & 48 & 2 & 2 & 2 & 2 & 100 & 100 & $t^{\prime}$ \\
\hline 6 & $\mathrm{~F}$ & 85 & Hand MCx & $\mathrm{R}$ & $\mathrm{C}$ & 1 & 0 & 0 & 1 & 1 & 100 & 100 & $--^{\prime}$ \\
\hline 7 & $\mathrm{~F}$ & 69 & $\mathrm{EC}+$ insular & $\mathrm{L}$ & SC & 3 & 2 & 0 & 2 & 2 & 90 & 100 & $-{ }^{\prime}$ \\
\hline 8 & $\mathrm{M}$ & 70 & MCA territory & $\mathrm{R}$ & $\mathrm{C}+\mathrm{SC}$ & 297 & 12 & 12 & 5 & 5 & 35 & 40 & $t^{\prime}$ \\
\hline 9 & $\mathrm{M}$ & 62 & EC & $\mathrm{L}$ & $\mathrm{C}+\mathrm{SC}$ & 5 & 1 & 1 & 3 & 2 & 85 & 100 & $-^{\prime}$ \\
\hline 10 & $\mathrm{~F}$ & 75 & Caud & $\mathrm{R}$ & SC & 5 & 2 & 1 & 3 & 3 & 91 & 100 & $-{ }^{\prime}$ \\
\hline 11 & $\mathrm{M}$ & 78 & Caud & $\mathrm{L}$ & SC & 10 & 2 & 1 & 2 & 1 & 100 & 100 & $+^{\prime}$ \\
\hline 12 & F & 73 & Thal & $\mathrm{L}$ & SC & 3 & 1 & 1 & 2 & 1 & 100 & 100 & $t^{\prime}$ \\
\hline 13 & $\mathrm{~F}$ & 48 & Thal & $\mathrm{L}$ & SC & 1 & 1 & 1 & 2 & 2 & 100 & 100 & $-{ }^{\prime}$ \\
\hline Mean & 66.8 & & & & & 32 & 2.5 & 2 & 2.2 & 1.9 & 90.4 & 94.6 & \\
\hline SD & 10.7 & & & & & 79 & 3.3 & 3.2 & 1 & 1.1 & 18.7 & 16.2 & \\
\hline Max & 85 & & & & & 297 & 12 & 12 & 5 & 5 & 100 & 100 & \\
\hline Min & 46 & & & & & 0.1 & 0 & 0 & 1 & 1 & 35 & 40 & \\
\hline
\end{tabular}

Sex: $\mathrm{M}=$ male; $\mathrm{F}=$ female. Age (years). $\mathrm{MCx}=$ motor cortex damage, $\mathrm{GB}=$ globus pallidus, $\mathrm{BGp}=$ basal ganglia posterior part, $\mathrm{MCA}$ territory $=$ extensive involvement of the middle cerebral artery territory, EC = external capsule, Caud = caudate, and Thal = thalamus. NIHSS score (maximum 42), Modified Rankin Scale (mRs) and Barthel Index (BI) at 1 and 3 months after stroke. Hemi $=$ hemisphere affected by stroke. $\mathrm{L}=$ left. $\mathrm{R}=$ right. Lesion classification: $\mathrm{C}=$ cortical, $\mathrm{S}=$ subcortical, and $\mathrm{C}+\mathrm{SC}=$ corticosubcortical lesion.

Lesion size (volume in $\mathrm{mm}^{3}$ ).

(event-related desynchronization; ERD), reflecting increased excitability, and increased at 500-1000 ms (event-related synchronization; ERS), reflecting removal of excitation [12, 13] or reduced excitability [14]. Inhibitory GABA-A agonist diazepam increases MEG beta activity $[15,16]$. Combined MEG and magnetic resonance spectroscopy showed a linear relationship between the beta ERS strength and GABA concentration [17]. Beta ERS has been shown to be significantly weaker in the $\mathrm{LH}$ than $\mathrm{NH}$; stronger ERS amplitude was correlated with a better affected hand function up to 3 months after stroke [18]. Reduction of beta ERS, however, correlated with clinical improvement after physiotherapy of patients with chronic stroke [19]. Movement-related beta ERD was significantly reduced in LH of stroke patients [20].

The hand representation in the somatosensory cortex (S1), estimated by somatosensory evoked fields (SEFs), is the largest one month after stroke and its increase was correlated with the affected hand function [21]. In TMS mapping, hand motor representation expands in the LH up to 1 month [22]. In animal models, somatosensory reorganization is activated immediately after the lesion by diminished GABA-A-related inhibition and by glutamatergic activation after one month [5].

We recorded both nTMS and MEG from patients during stroke recovery. We hypothesized to see correlations between ERD and MT related to the early cortical excitability and between ERS and SICI, both attributed to GABA-A-related processes. Moreover, we expected that ICF, reflecting glutamatergic activity, would correlate with the somatosensory modifications in MEG, as glutamate is associated with late somatosensory plasticity [5].

\section{Methods}

MEG and nTMS were recorded from thirteen patients (age $67.3 \pm 11$ years, 8 women), with first-ever ischemic stroke in the middle cerebral artery territory one (T1) and three months after stroke (T2). Hand function was impaired in all patients. Six patients had a subcortical and seven patients had a subcorticocortical or cortical stroke (Table 1). At T1, one patient used amitriptyline $10 \mathrm{mg} /$ day and another used citalopram $10 \mathrm{mg} /$ day. One patient used zopiclone $7.5 \mathrm{mg}$ for occasional sleeplessness. At T2, citalopram $10 \mathrm{mg} /$ day was used by one patient. Other patients did not receive drugs known to modify cortical excitability. The ethical committee of the Hospital District of Helsinki and Uusimaa approved the project. Data from these patients has been presented previously $[18,21,23,24]$. As the patients having both TMS and MEG recordings are a subset of the previous patient groups, we recalculated the group-level electrophysiological parameters to show that the present patient group is sufficiently similar to those reported previously (see Supplementary Tables 1 and 2 in Supplementary Material available online at http://dx.doi.org/10.1155/2015/309546). Only the features needed for interpretation of the present results are described.

2.1. nTMS Measurements. An eXimia navigated magnetic stimulator with a coplanar figure-of-eight coil of $70 \mathrm{~mm}$ wing radius (Nexstim Ltd., Helsinki, Finland) was used for nTMS. Patients' MRIs, required for navigation, were scanned at T1 and used also at $\mathrm{T} 2$.

The site eliciting the largest MEP amplitude in first dorsal interosseous muscle was located. The resting MT was 
determined from this site as described in [25]. The site was then stimulated by single TMS pulses at $110 \%$ of MT and by the PP-TMS at $90 \%$ for conditioning and $110 \%$ of MT for test pulses. Fifteen single pulses or pairs of conditioning and test pulses were delivered in each stimulation session. The interval between stimuli was $3.3 \mathrm{~s}$ and the intersession interval varied between 1 and $3 \mathrm{~min}$. The ISI selected for the paired-pulse stimuli was $2 \mathrm{~ms}$ for SICI and $12 \mathrm{~ms}$ for ICF. The peak-to-peak amplitudes of MEPs elicited by PP-TMS were normalized by dividing them by the corresponding single-pulse MEP amplitude to simplify subject-to-subject comparisons [6].

2.2. MEG Recordings. MEG during rest and tactile stimulation of the thumb (D1), index (D2), and little finger (D5) were recorded with a 306-sensor neuromagnetometer (Elekta Neuromag, Helsinki, Finland) in BioMag laboratory, right before the nTMS measurement. The interfering signals were suppressed by Maxfilter software [26]. The signals were filtered through $0.03-308 \mathrm{~Hz}$ and digitized at $941 \mathrm{~Hz}$.

Time-frequency representations (TFR [27]) in the 10$30 \mathrm{~Hz}$ band were calculated to define the frequency range of beta modulation, which was quantified by the temporal spectral evolution method (TSE [10]) from signals of 2 to 4 MEG sensors showing the strongest reactivity. Only the contralateral beta modifications (the affected hand stimulation for the $\mathrm{LH}$ and the unaffected hand for $\mathrm{NH}$ ) were analyzed. Onset and offset of the ERD and ERS were defined as a time point when the signal differed 2 SDs from the baseline. The absolute ERD and ERS strengths were calculated from the peak amplitudes and converted into relative values in relation to the $300 \mathrm{~ms}$ prestimulus baseline [18].

For SEFs, about 120 responses were averaged for stimulation of D2 (ISI $3005 \mathrm{~ms}$ ), and D1 and D5 (ISI $1005 \mathrm{~ms}$ ) in separate sessions. The size of the hand representation in the SI was determined by calculating the Euclidean distance in $x y z$-space between the equivalent current dipoles (ECDs) of the earliest responses to D1 and D5 stimulation [21]. The amplitudes of SEFs to D2 stimulation [23] were used in the analyses.

SPSS 14.0 software was employed for statistical analysis (SPSS Inc., Chicago, Illinois, USA). Spearman's correlation coefficients were calculated between nTMS and MEG parameters. Multiple comparison correction was carried out according to the number of tests $(N=32)$ suggested by the four prior hypotheses (T1 and T2 were tested separately; $\mathrm{LH}$ and $\mathrm{NH}$ variations lead to four tests in each case; $N=$ $4 * 4 * 2=32$ ). The significance level was set at $p<0.05$. We also present significances of correlation values without multiple comparisons and supply all correlation coefficients and corresponding $p$ values (cf. $[1,9,28]$ for a similar approach and [29] for its statistical aspects). The differences between nTMS and MEG values obtained at T1 and T2 were tested with Student's $t$-test.

\section{Results}

3.1. Navigated TMS. In the LH, MEPs were found in 11 patients both at $\mathrm{T} 1$ and at $\mathrm{T} 2$ and were present in the $\mathrm{NH}$ in all patients. Group average MTs did not change between measurements or hemispheres. Individual MT values are given in Supplementary Table 1. MT was higher for LH than $\mathrm{NH}$ in 9 patients at T1 $(p<0.05$, binomial test) and in 10 patients at T2 ( $p<0.05$, binomial test). The MTs in the LH and NH correlated strongly both at $\mathrm{T} 1(r=.82, p<.01)$ and at $\mathrm{T} 2(r=.78, p<.01)$.

PP2ms stimulation of LH inhibited MEPs in 7 patients at both T1 and T2. PP2ms stimulations of NH did not inhibit MEPs (disinhibition; diminished SICI) in 5 patients at $\mathrm{T} 1$ and in 3 patients at T2. SICI values did not correlate significantly between the hemispheres at $\mathrm{T} 1$ or $\mathrm{T} 2$.

PP12ms stimulation of LH facilitated MEPs (ICF) in 10 out of 11 patients at $\mathrm{T} 1$ and in all patients at $\mathrm{T} 2$. In NH, ICF was induced in all patients at T1. At T2, ICF was not observed in 4 patients. MEP amplitudes elicited by PP12ms stimulations were correlated between the $\mathrm{LH}$ and $\mathrm{NH}$ at $\mathrm{T} 1(r=.62 ; p<$ .05 ) but not at T2 (Supplementary Table 2).

3.2. MEG. ERD started $140 \pm 10 \mathrm{~ms}$ after tactile stimulation and peaked at $250 \pm 10 \mathrm{~ms}$. The subsequent ERS started at $520 \pm 40 \mathrm{~ms}$ and peaked at $900 \pm 70 \mathrm{~ms}$. At Tl, ERD was absent in one patient and ERS in two patients in the LH; ERS was missing from the $\mathrm{NH}$ in one patient. At T2, the ERD was present in every LH whereas ERS was absent in two. Both were present in all NHs. ERDs did not differ between the hemispheres. ERS was smaller in the LH than NH at T1 ( $46 \pm 31 \%$ versus $63 \pm 32 \%$; $p<.05$ ); at T2, the difference was nonsignificant. SEFs from both hemispheres were detected in 12 patients at $\mathrm{T} 1$ and in all patients at $\mathrm{T} 2$. They were smaller in the $\mathrm{LH}$ than $\mathrm{NH}$ at $\mathrm{T} 1$ ( $25 \mathrm{nAm}$ versus $32 \mathrm{nAm} ; p<.04)$ but not at T2. The SI hand representation area was larger in the $\mathrm{LH}$ than $\mathrm{NH}$ at $\mathrm{T} 1(12 \pm 3 \mathrm{~mm}$ versus $10 \pm 3 \mathrm{~mm} p<.003)$ but not at T2 (Supplementary Table 3).

3.3. Correlations between the nTMS and MEG. We tested the correlations indicated by the selected hypotheses. The plots of the most relevant correlations are depicted in Figure 1 to show that they were not driven by outliers. All correlations are displayed in Table 2 to enable evaluation of significance of our hypotheses against general effects of the lesions.

The MT and ERD were correlated in the LH at T1 $(r=$ $-.66, p<.03)$, indicating that small ERD was associated with a high MT (Figure 1(a)). At T2, this correlation was weaker $(r=-.58, p<.06)$. However, the MT of the LH correlated with the ERD of NH $(r=-.62, p<.04)$, and the MT of the NH correlated with ERD of the LH $(r=-.65, p<.02)$, suggesting that high MT was associated with a small ERD in the opposite hemisphere at T2 (Table 2).

SICI and the ERS did not correlate at $\mathrm{T} 1$ or in $\mathrm{LH}$ at T2. In the NH, high ERS was associated with a strong SICI $(r=-.59, p<.04$; Figure 1(b)). In addition to hypothesized correlations, SICI of the $\mathrm{NH}$ and ERD of the $\mathrm{LH}$ at T2 were correlated $(r=-.82, p<.001)$, indicating that strong ERD in the LH was associated with a strong SICI in the NH. SICI in the LH was correlated also with the SI amplitude of the LH $(r=-.64, p<.04)$, indicating that small SI amplitude was associated with a weak SICI (Table 2). 


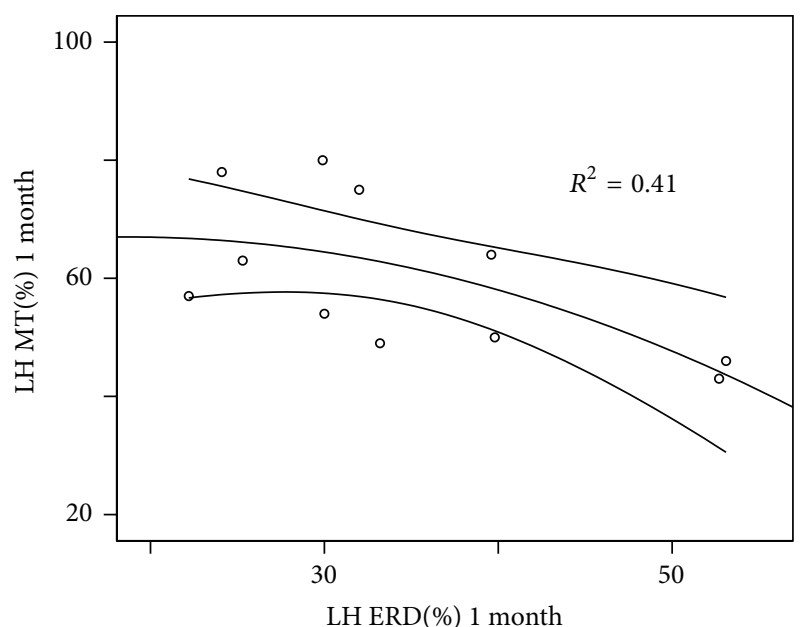

(a)

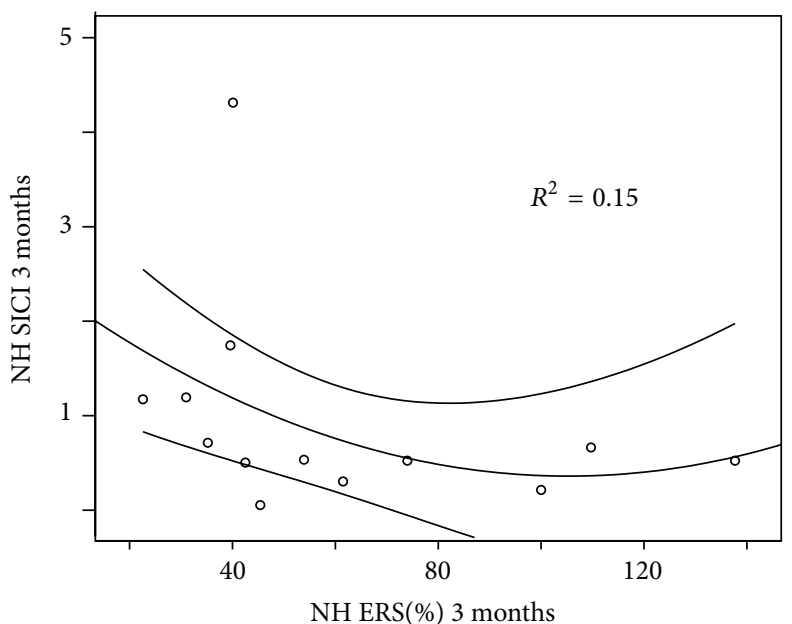

(b)

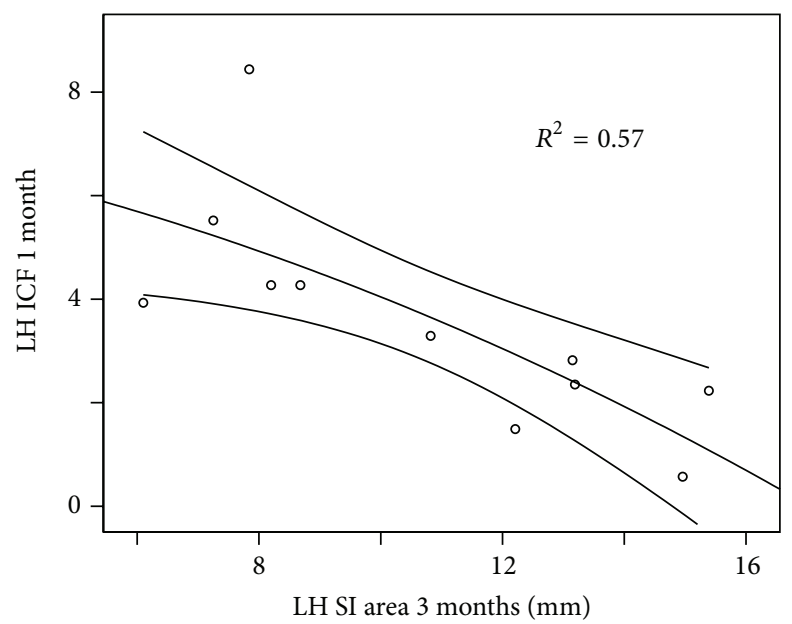

(c)

FIGURE 1: Scatterplots, quadratic fits, and 95\% confidence intervals of the motor thresholds and ERD values in the lesioned hemisphere at 1 month (a), SICI and ERS values in the nonlesioned hemisphere at 3 months (b), and ICF values at 1 month and SI area at 3 months in the lesioned hemisphere (c).

ICF and SEF parameters did not correlate at T1. At T2, ICF in the LH correlated with the S1 amplitude of LH ( $r=$ $-.65, p<.03)$; if ICF was strong, the SI amplitude was small. In addition, ICF in the NH correlated $(r=-.82$, $p<.001$ ) with the SI finger representation area of the LH; this correlation remained significant also after Bonferroni correction (Table 2). Moreover, ICF in the LH at T1 was correlated $(r=-.83 ; p<.002)$ with the SI finger representation area of $\mathrm{LH}$ at $\mathrm{T} 2$; high ICF at T1 resulted in a small hand representation area at T2 (Figure $1(\mathrm{c})$ ).

\section{Discussion}

Our study is the first to compare MEG and nTMS excitability parameters during stroke recovery. Navigated TMS, not applied previously in longitudinal studies of stroke patients, enabled the precise replication of the stimulus site between separate measurements, adding reliability to the follow-up.
We found correlations between cortical excitability estimates derived from nTMS and MEG.

As expected, we found correlations between MT and ERD, but in only one of the four possible intrahemispheric and two out of four interhemispheric conditions. The SICI and beta ERS, both attributed to GABAergic mechanisms, were correlated in one of their four possible intrahemispheric conditions (in the $\mathrm{NH}$ at T2). Interhemispheric correlations of SICI were not limited to ERS. One reason for this may be that various GABA-A receptor subtypes contribute to SICI. Nonselective GABA-A receptor activators modify SICI whereas the GABA-A1 receptor specific zolpidem did not [7]. Nonselective GABA-A agonist zopiclone increased MEG beta activity whereas zolpidem suppressed beta activity in the vicinity of stroke lesion [30]. Moreover, in healthy subjects, diazepam increased MEG ERD but did not affect ERS when the increase of baseline beta activity was taken into account [16]. This nonspecificity could contribute to the correlations of SICI with the ERD and MT as well. 
TABLE 2: Spearman correlations between the nTMS and MEG parameters at 1 month $(1 \mathrm{mo})$ and three months $(3 \mathrm{mo})$ after the stroke. The correlations between event-related desynchronization (ERD) and motor threshold (MT), event-related synchronization (ERS) and shortinterval cortical inhibition (SICI), and intracortical facilitation (ICF) and somatosensory evoked field source strength (SI) and somatosensory hand representation area (SIhr), aligned with hypotheses, are depicted in bold font. ${ }^{*}$ Significance of $p<.05$; ${ }^{* *}$ significance of $p<.01$ without multiple comparison correction; ${ }^{* *}$ (marked with bold italic) statistical significance $(p<.05)$ with multiple comparison correction (Bonferroni) for $N=32$.

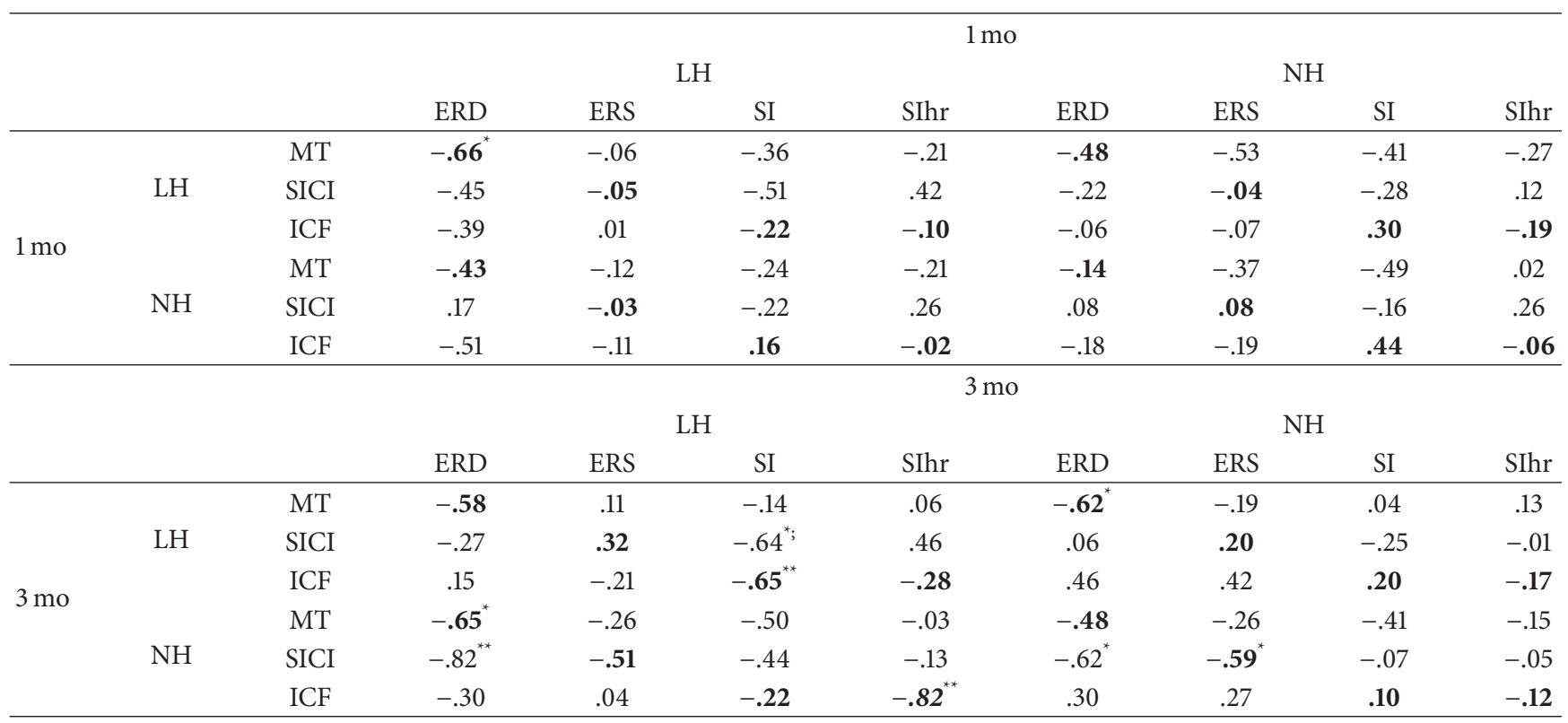

Correlations between nTMS parameters and MEG ERD/ERS were stronger at T2 than at T1. Analogously, most TMS intracortical excitability measures did not correlate with the hand function acutely but did so 3 months after stroke [1]. Recovery of sensorimotor fMRI activation to digit stimulation from 1 to 3 months was correlated with final motor function [31], emphasizing the importance of this time period for stroke recovery.

ICF correlated with SI hand area size at T2. As ICF is attributed mainly to glutaminergic mechanisms, glutamate may contribute to stroke-induced plasticity. Somewhat surprisingly, high ICF at T1 correlated with small SI hand area at T2 (Figure 1(c)); thus, the narrowing towards normal hand representation size may be supported by glutaminergic activity. ICF did not correlate with the MEG ERD/ERS, and the SI hand area and beta ERD/ERS were not correlated [18]; this suggests different mechanisms underlying SICI and ICF (see [7] for a detailed discussion).

Several correlations emphasized interhemispheric connectivity (see Figure 2 and Table 2). For example, high MT was associated with a small ERD in the opposite hemisphere, and strong ERD in the LH was associated with a strong SICI in the NH. This suggests that the hemispheric balance of excitability is important in stroke recovery. Dexterity is impaired in both hands after unilateral subcortical middle cerebral artery stroke. Increased excitability within the unaffected motor cortex may cause imbalance between the homologous cortical motor areas and worsen also the ipsilesional hand coordination (for references, see [32]). MTs between the hemispheres were strongly correlated both at
$\mathrm{T} 1$ and at $\mathrm{T} 2$. Thus, some functional correlations may relate to the modified general excitability properties of the motor system, instead of effects in the immediate vicinity of the stroke [28].

Correlations between MEG and TMS parameters of cortical excitability were relatively loose. Several factors may explain this feature. TMS results give direct information of the changes in the motor output and the immediate effects of TMS are relatively local. However, also subcortical and spinal processes affect the MEPs used to evaluate the TMS effects. MEG reveals the activity of the whole cortical mantle and enables mapping of network effects generated by stroke. MEG source analysis suggests mainly motor cortex origin of beta ERS $[13,33,34]$. However, in electrocorticography, recorded directly from the cortex, beta ERD and ERS appear outside of pre- and postcentral gyri [35], in supplementary motor cortex [36], or broadly from pre- and postcentral gyri, frontolateral and medial cortex $[37,38]$. The widespread cortical generation of the ERD and ERS may make them resilient to small cortical strokes. Multitude of generators may contribute to considerable variability of source locations of beta ERD in stroke patients (cf. [20]). Multiple sources underlying MEG signals may also explain resilience of auditory evoked fields after small strokes [39]. Stronger correlations between ICF and SI parameters than between MT and SICI and ERS/ERD may, in part, result from spatially more limited source areas of $\mathrm{S} 1$ responses than those of ERD/ERS. It can be expected that MEG and nTMS produce complementary information about the effects of stroke on cortical networks. Moreover, MEG parameters in the affected hemisphere and nTMS indices in 

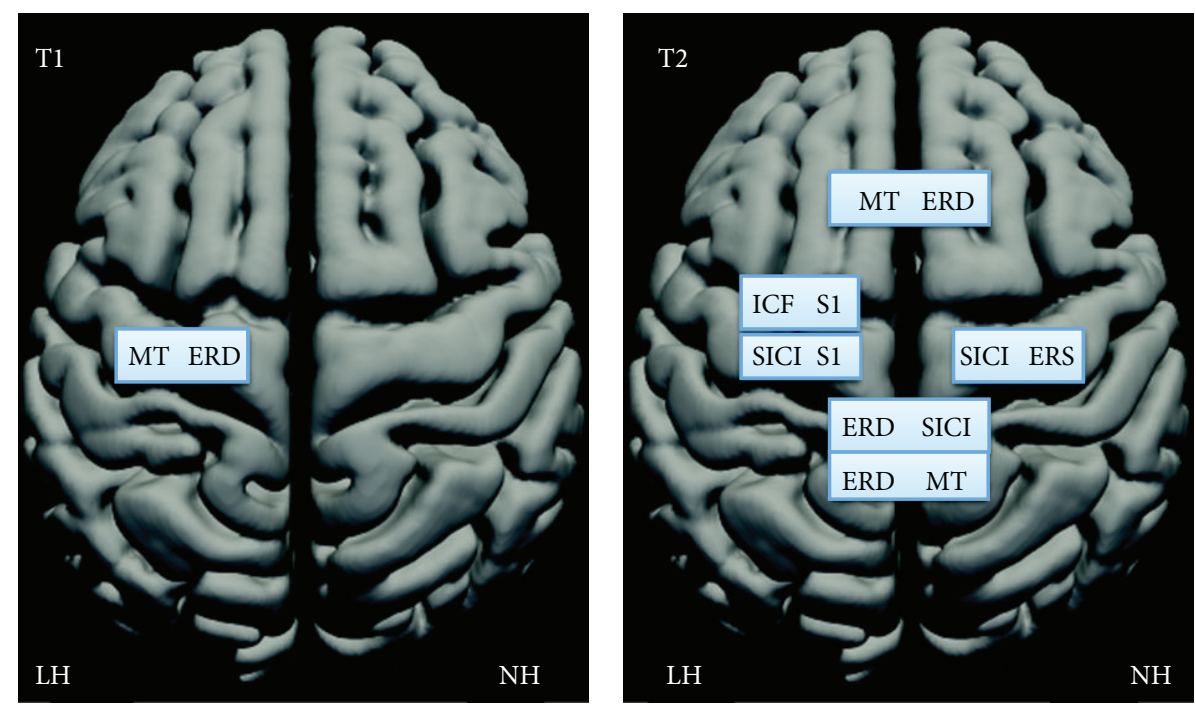

FIGURE 2: TMS and MEG parameters correlating at T1 (one month) and at T2 (three months), drawn on a schematic brain with lesioned (LH) and nonlesioned hemisphere (NH). The intrahemispheric connections are drawn on one hemisphere and interhemispheric connections on both hemispheres. Note strong increase of both intra- and interhemispheric correlations from one to three months after the stroke.

the unaffected hemisphere were correlated with the motor performance of the affected hand (cf. $[18,21,24]$ ). This emphasizes the importance of combining these two methods.

ERD in the $8-22 \mathrm{~Hz}$ band may reflect downregulation of intracortical inhibition in the human motor cortex, as TMS delivered during ERD is associated with increased MEP amplitudes and reduced SICI [40]. However, $1 \mathrm{~Hz}$ repetitive TMS over the motor cortex reduces MEPs to subsequent single TMS pulses, indicating inhibition, but decreases postmovement beta ERS [41], and intermittent theta burst TMS facilitates MEPs but increases postmovement beta ERS [42]. Beta ERS is reduced in patients with myoclonus epilepsy, indicating increased cortical excitability [43]. In line, SICI is decreased in myoclonus epilepsy patients; however, MT is increased [44, 45], and the silent period after the TMS pulse, reflecting motor cortical postsynaptic inhibition [7], is prolonged, indicating prevailing inhibitory cortical tonus [45]. Thus, only some aspects of the cortical excitability may be shared in excitability estimates obtained by TMS and MEG.

Our results suggest that some TMS and MEG excitability measures reflect the activity of the same transmitter systems. However, high MT and absence of ERD/ERS may also correlate because of severely affected sensorimotor connections between the periphery and the cortex. We detected SEFs in 12/13 patients and MEPs in 11/13 patients already at T1, indicating that both somatosensory and motor pathways were conveying signals. Motor function can be maintained despite significant damage to the corticospinal tract, as estimated from MT of stroke patients [1]. Moreover, we observed fewer correlations at $\mathrm{T} 1$, when the sensorimotor pathways probably were more affected, than at T2. Large lesions may create spurious correlations between the excitability parameters within LH. However, such spurious correlations should remain stable or decrease during recovery from $\mathrm{T} 1$ to $\mathrm{T} 2$ but not increase, as in our data.

The limitations of our study include the small size of the patient group as the precise features of structural and functional changes may differ among the patients. Cortical excitability is modified differently in cortical and subcortical strokes $[46,47]$. This, however, should not alter our correlations between the TMS and MEG, as both were recorded from the same patients. Possible effects of medication on excitability should go in parallel for MEG and nTMS, as the patients were tested sequentially during the same day. The patients were not tested in the acute stage with TMS, and MEG recordings showed most dramatic ERD and ERS modifications between the acute phase and T1 [18]. Although MT in the $\mathrm{LH}$ is correlated with the paretic hand function in acute stroke, this correlation, however, weakens during recovery, and TMS intracortical excitability parameters correlated with the clinical performance best at 3 months [1]. Longer followup could have produced additional correlations. The $2 \mathrm{~ms}$ or $12 \mathrm{~ms}$ ISIs, selected for our paired-pulse stimuli, produce clear SICI and ICF in healthy subjects [48] and in stroke patients [28], but we did not test other parameters, which could have produced stronger correlations between nTMS and MEG.

\section{Conclusions}

ICF and SI response amplitude and area size, MT and the ERD of the hemisphere harboring the stroke lesion, and SICI and ERS of the nonlesioned hemisphere are correlated in stroke patients. Numerous correlations of the excitability parameters between the $\mathrm{LH}$ and $\mathrm{NH}$ emphasize the importance of the hemispheric balance of the excitatoryinhibitory properties of the sensorimotor system in analyzing the stroke-related dysfunction during stroke recovery. 


\section{Conflict of Interests}

The authors declare that there is no conflict of interests regarding the publication of this paper.

\section{Acknowledgments}

The authors thank Suvi Heikkilä and Jari Kainulainen for their help in the recordings, Jussi Nurminen for help in the data analysis, and the ergo- and physiotherapists at the Department of Neurology, HUCH, for help in the clinical testing of the patients. Dr. Ilkka Nissilä gave valuable advice about statistics. The study was financially supported by the Academy of Finland (National Centers of Excellence Program 2006-2011), the Helsinki University Central Hospital Research Fund, and the SalWe Research Program for Mind and Body (Tekes, the Finnish Funding Agency for Technology and Innovation Grant 1104/10).

\section{References}

[1] O. B. C. Swayne, J. C. Rothwell, N. S. Ward, and R. J. Greenwood, "Stages of motor output reorganization after hemispheric stroke suggested by longitudinal studies of cortical physiology," Cerebral Cortex, vol. 18, no. 8, pp. 1909-1922, 2008.

[2] N. Dancause and R. J. Nudo, "Shaping plasticity to enhance recovery after injury," Progress in Brain Research, vol. 192, pp. 273-295, 2011.

[3] C. Grefkes and G. R. Fink, "Connectivity-based approaches in stroke and recovery of function," The Lancet Neurology, vol. 13, no. 2, pp. 206-216, 2014.

[4] R. J. Nudo, B. M. Wise, F. SiFuentes, and G. W. Milliken, "Neural substrates for the effects of rehabilitative training on motor recovery after ischemic infarct," Science, vol. 272, no. 5269, pp. 1791-1794, 1996.

[5] P. E. Garraghty, L. L. Arnold, C. L. Wellman, and T. M. Mowery, "Receptor autoradiographic correlates of deafferentationinduced reorganization in adult primate somatosensory cortex," Journal of Comparative Neurology, vol. 497, no. 4, pp. 636-645, 2006.

[6] J. Liepert, F. Hamzei, and C. Weiller, "Motor cortex disinhibition of the unaffected hemisphere after acute stroke," Muscle and Nerve, vol. 23, no. 11, pp. 1761-1763, 2000.

[7] U. Ziemann, "Pharmaco-transcranial magnetic stimulation studies of motor excitability," in Handbook of Clinical Neurology, A. M. Lozano and M. Hallet, Eds., vol. 116 of Brain Simulation, Elsevier, 2013.

[8] P. Talelli, R. J. Greenwood, and J. C. Rothwell, "Arm function after stroke: neurophysiological correlates and recovery mechanisms assessed by transcranial magnetic stimulation," Clinical Neurophysiology, vol. 117, no. 8, pp. 1641-1659, 2006.

[9] U. Takechi, K. Matsunaga, R. Nakanishi et al., "Longitudinal changes of motor cortical excitability and transcallosal inhibition after subcortical stroke," Clinical Neurophysiology, vol. 125, no. 10, pp. 2055-2069, 2014.

[10] R. Salmelin and R. Hari, "Spatiotemporal characteristics of sensorimotor neuromagnetic rhythms related to thumb movement," Neuroscience, vol. 60, no. 2, pp. 537-550, 1994.

[11] R. Enatsu, T. Nagamine, J. Matsubayashi et al., "The modulation of rolandic oscillation induced by digital nerve stimulation and self-paced movement of the finger: a MEG study," Journal of the Neurological Sciences, vol. 337, no. 1-2, pp. 201-211, 2014.

[12] G. Pfurtscheller and F. H. L. da Silva, "Event-related EEG/MEG synchronization and desynchronization: basic principles," Clinical Neurophysiology, vol. 110, no. 11, pp. 1842-1857, 1999.

[13] R. Salmelin, M. Hämäläinen, M. Kajola, and R. Hari, "Functional segregation of movement-related rhythmic activity in the human brain," NeuroImage, vol. 2, no. 4, pp. 237-243, 1995.

[14] R. Chen, B. Corwell, and M. Hallett, "Modulation of motor cortex excitability by median nerve and digit stimulation," Experimental Brain Research, vol. 129, no. 1, pp. 77-86, 1999.

[15] O. Jensen, P. Goel, N. Kopell, M. Pohja, R. Hari, and B. Ermentrout, "On the human sensorimotor-cortex beta rhythm: sources and modeling," NeuroImage, vol. 26, no. 2, pp. 347-355, 2005.

[16] S. D. Hall, I. M. Stanford, N. Yamawaki et al., "The role of GABAergic modulation in motor function related neuronal network activity," NeuroImage, vol. 56, no. 3, pp. 1506-1510, 2011.

[17] W. Gaetz, J. C. Edgar, D. J. Wang, and T. P. L. Roberts, "Relating MEG measured motor cortical oscillations to resting $\gamma$-aminobutyric acid (GABA) concentration," NeuroImage, vol. 55, no. 2, pp. 616-621, 2011.

[18] K. Laaksonen, E. Kirveskari, J. P. Mäkelä et al., "Effect of afferent input on motor cortex excitability during stroke recovery," Clinical Neurophysiology, vol. 123, no. 12, pp. 2429-2436, 2012.

[19] T. W. Wilson, A. Fleischer, D. Archer, S. Hayasaka, and L. Sawaki, "Oscillatory MEG motor activity reflects therapyrelated plasticity in stroke patients," Neurorehabilitation and Neural Repair, vol. 25, no. 2, pp. 188-193, 2011.

[20] H. E. Rossiter, M.-H. Boudrias, and N. S. Ward, "Do movementrelated beta oscillations change following stroke?" Journal of Neurophysiology, vol. 112, pp. 2053-2058, 2014.

[21] K. Roiha, E. Kirveskari, M. Kaste et al., "Reorganization of the primary somatosensory cortex during stroke recovery," Clinical Neurophysiology, vol. 122, no. 2, pp. 339-345, 2011.

[22] G. F. Wittenberg, E. P. Bastings, C. Scales, and D. Good, "Evolution of TMS motor maps during recovery after stroke," NeuroImage, vol. 13, supplement, no. 6, 1281 pages, 2001.

[23] N. Forss, S. Mustanoja, K. Roiha et al., "Activation in parietal operculum parallels motor recovery in stroke," Human Brain Mapping, vol. 33, no. 3, pp. 534-541, 2012.

[24] P. Lioumis, S. Mustanoja, R. Bikmullina et al., "Probing modifications of cortical excitability during stroke recovery with navigated TMS," Topics in Stroke Rehabilitation, vol. 19, pp. 182192, 2012.

[25] P. M. Rossini, A. T. Barker, A. Berardelli et al., "Non-invasive electrical and magnetic stimulation of the brain, spinal cord and roots: basic principles and procedures for routine clinical application. Report of an IFCN committee," Electroencephalography and Clinical Neurophysiology, vol. 91, no. 2, pp. 79-92, 1994.

[26] S. Taulu and J. Simola, "Spatiotemporal signal space separation method for rejecting nearby interference in MEG measurements," Physics in Medicine and Biology, vol. 51, no. 7, pp. 17591768,2006

[27] C. Tallon-Baudry, O. Bertrand, C. Wienbruch, B. Ross, and C. Pantev, "Combined EEG and MEG recordings of visual $40 \mathrm{~Hz}$ responses to illusory triangles in human," NeuroReport, vol. 8, no. 5, pp. 1103-1107, 1997.

[28] G. F. Wittenberg, E. P. Bastings, A. M. Fowlkes, T. M. Morgan, D. C. Good, and T. P. Pons, "Dynamic course of intracortical TMS paired-pulse responses during recovery of motor function after 
stroke," Neurorehabilitation and Neural Repair, vol. 21, no. 6, pp. 568-573, 2007.

[29] T. V. Perneger, "What's wrong with Bonferroni adjustments," The British Medical Journal, vol. 316, no. 7139, pp. 1236-1238, 1998.

[30] S. D. Hall, N. Yamawaki, A. E. Fisher, R. P. Clauss, G. L. Woodhall, and I. M. Stanford, "GABA(A) alpha-1 subunit mediated desynchronization of elevated low frequency oscillations alleviates specific dysfunction in stroke-a case report," Clinical Neurophysiology, vol. 121, no. 4, pp. 549-555, 2010.

[31] J. D. Schaechter, C. A. M. M. van Oers, B. N. Groisser et al., "Increase in sensorimotor cortex response to somatosensory stimulation over subacute poststroke period correlates with motor recovery in hemiparetic patients," Neurorehabilitation and Neural Repair, vol. 26, no. 4, pp. 325-334, 2012.

[32] D. A. Nowak, C. Grefkes, M. Dafotakis, J. Küst, H. Karbe, and G. R. Fink, "Dexterity is impaired at both hands following unilateral subcortical middle cerebral artery stroke," European Journal of Neuroscience, vol. 25, no. 10, pp. 3173-3184, 2007.

[33] M. T. Jurkiewicz, W. C. Gaetz, A. C. Bostan, and D. Cheyne, "Post-movement beta rebound is generated in motor cortex: evidence from neuromagnetic recordings," NeuroImage, vol. 32, no. 3, pp. 1281-1289, 2006

[34] W. Gaetz and D. Cheyne, "Localization of sensorimotor cortical rhythms induced by tactile stimulation using spatially filtered MEG," NeuroImage, vol. 30, no. 3, pp. 899-908, 2006.

[35] N. E. Crone, D. L. Miglioretti, B. Gordon et al., "Functional mapping of human sensorimotor cortex with electrocorticographic spectral analysis: I. Alpha and beta event-related desynchronization," Brain, vol. 121, no. 12, pp. 2271-2299, 1998.

[36] S. Ohara, A. Ikeda, T. Kunieda et al., "Movement-related change of electrocorticographic activity in human supplementary motor area proper," Brain, vol. 123, no. 6, pp. 1203-1215, 2000.

[37] W. Szurhaj, P. Derambure, E. Labyt et al., "Basic mechanisms of central rhythms reactivity to preparation and execution of a voluntary movement: a stereoelectroencephalographic study," Clinical Neurophysiology, vol. 114, no. 1, pp. 107-119, 2003.

[38] D. Sochůrková, I. Rektor, P. Jurák, and A. Stančák, "Intracerebral recording of cortical activity related to self-paced voluntary movements: a Bereitschaftspotential and event-related desynchronization/synchronization. SEEG study," Experimental Brain Research, vol. 173, no. 4, pp. 637-649, 2006.

[39] J. P. Mäkelä, R. Hari, L. Valanne, and A. Ahonen, "Auditory evoked magnetic fields after ischemic brain lesions," Annals of Neurology, vol. 30, no. 1, pp. 76-82, 1991.

[40] M. Takemi, Y. Masakado, M. Liu, and J. Ushiba, "Eventrelated desynchronization reflects downregulation of intracortical inhibition in human primary motor cortex," Journal of Neurophysiology, vol. 110, no. 5, pp. 1158-1166, 2013.

[41] Y. Tamura, M. Hoshiyama, H. Nakata et al., "Functional relationship between human rolandic oscillations and motor cortical excitability: an MEG study," European Journal of Neuroscience, vol. 21, no. 9, pp. 2555-2562, 2005.

[42] Y.-F. Hsu, K.-K. Liao, P.-L. Lee et al., "Intermittent theta burst stimulation over primary motor cortex enhances movementrelated beta synchronisation," Clinical Neurophysiology, vol. 122, no. 11, pp. 2260-2267, 2011.

[43] T. Silén, N. Forss, O. Jensen, and R. Hari, "Abnormal reactivity of the $\sim 20-\mathrm{Hz}$ motor cortex rhythm in unverricht lundborg type progressive myoclonus epilepsy," NeuroImage, vol. 12, no. 6, pp. 707-712, 2000.
[44] P. Brown, M. C. Ridding, K. J. Werhahn, J. C. Rothwell, and C. D. Marsden, "Abnormalities of the balance between inhibition and excitation in the motor cortex of patients with cortical myoclonus," Brain, vol. 119, no. 1, pp. 309-317, 1996.

[45] N. Danner, P. Julkunen, J. Khyuppenen et al., "Altered cortical inhibition in Unverricht-Lundborg type progressive myoclonus epilepsy (EPM1)," Epilepsy Research, vol. 85, no. 1, pp. 81-88, 2009.

[46] J. Liepert, C. Restemeyer, T. Kucinski, S. Zittel, and C. Weiller, "Motor strokes: the lesion location determines motor excitability changes," Stroke, vol. 36, no. 12, pp. 2648-2653, 2005.

[47] M. Ameli, C. Grefkes, F. Kemper et al., "Differential effects of high-frequency repetitive transcranial magnetic stimulation over ipsilesional primary motor cortex in cortical and subcortical middle cerebral artery stroke," Annals of Neurology, vol. 66, no. 3, pp. 298-309, 2009.

[48] L. Säisänen, P. Julkunen, E. Niskanen et al., "Short- and intermediate-interval cortical inhibition and facilitation assessed by navigated transcranial magnetic stimulation," Journal of Neuroscience Methods, vol. 195, no. 2, pp. 241-248, 2011. 

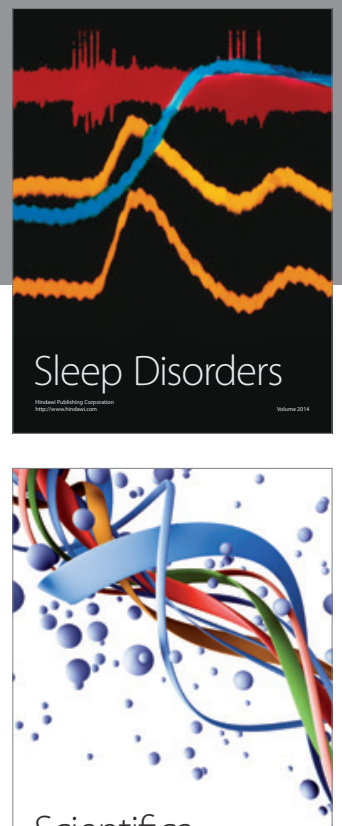

Scientifica
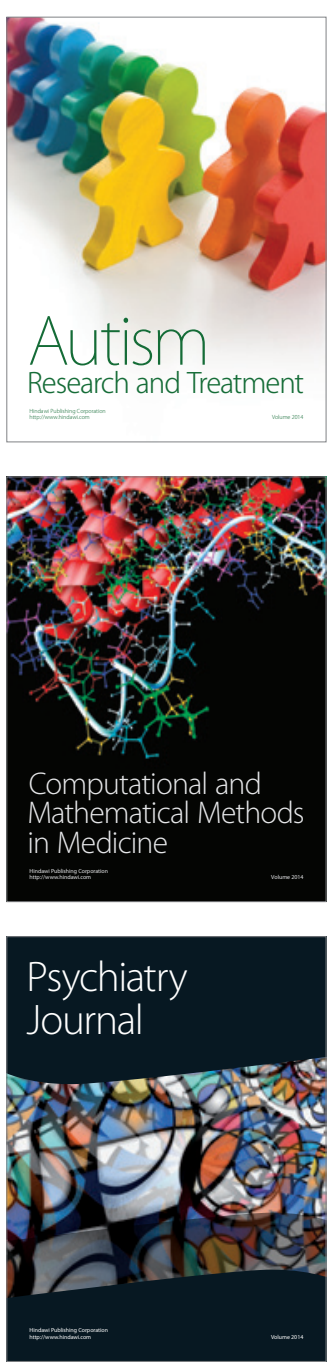
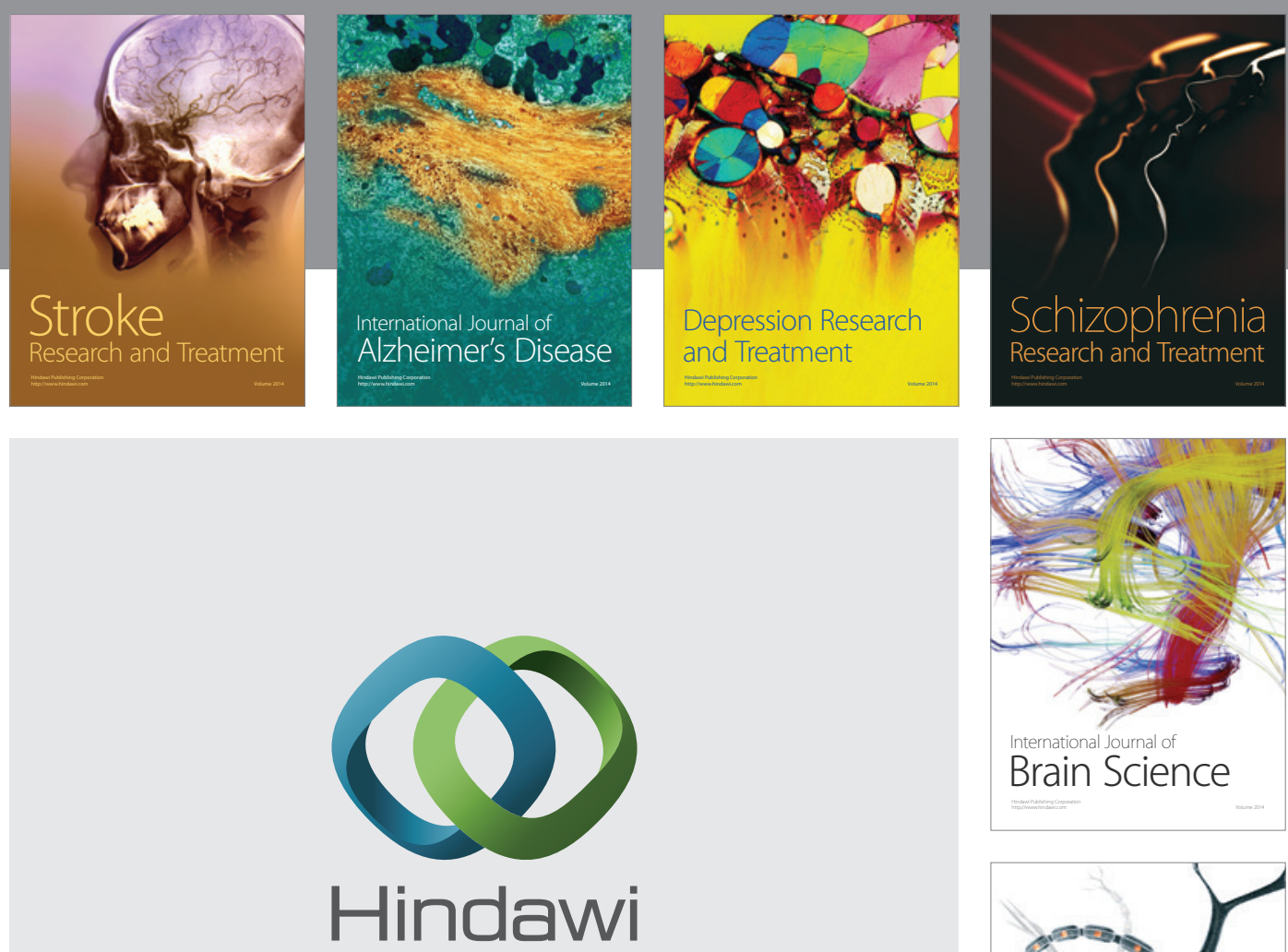

Submit your manuscripts at

http://www.hindawi.com
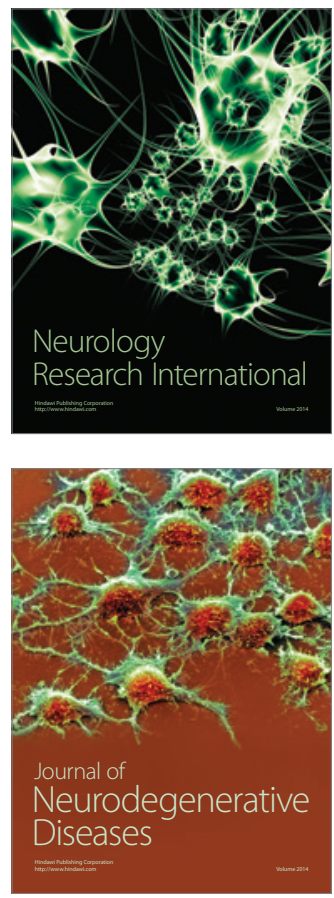

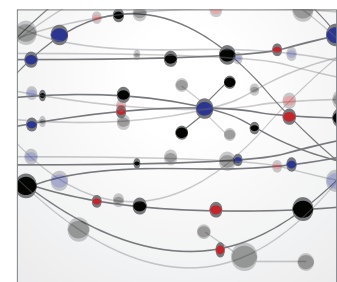

The Scientific World Journal
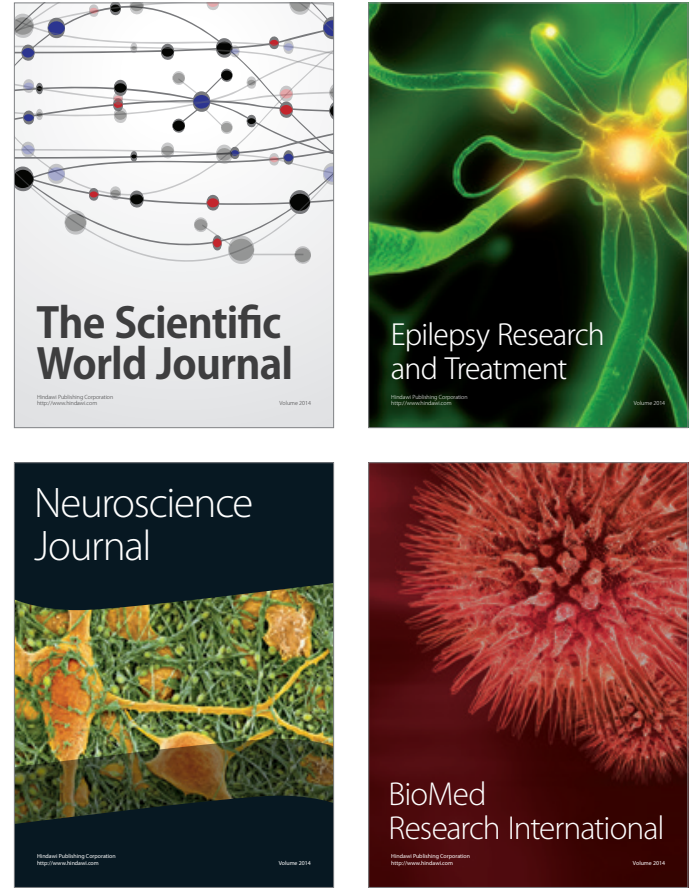

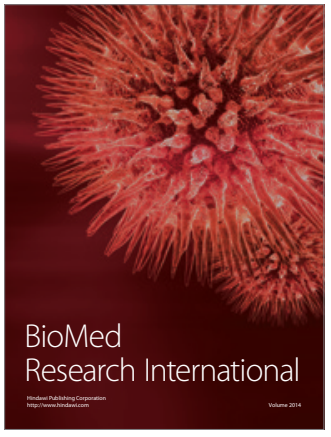

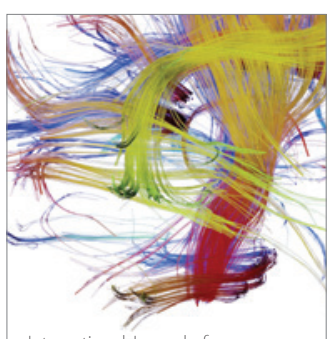

Brain Science

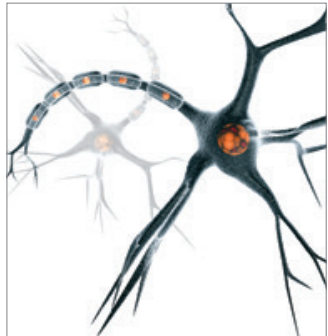

Neural Plasticity
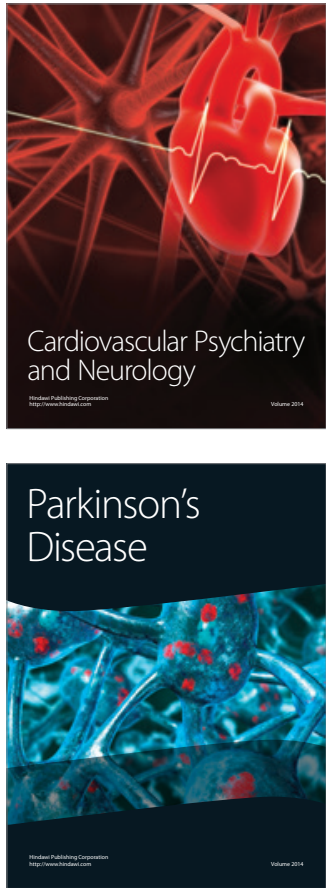